\title{
Nutzfahrzeug als Impulsgeber
}

Liebe Leserin, lieber Leser,

in der Öffentlichkeit wenig beachtet, ist die Relevanz der IAA Nutzfahrzeuge für die Zielgruppe dennoch sehr hoch. Die aktuelle ATZ trägt dem in ihrer Titelstrecke Rechnung und berichtet zum Beispiel über das EU-Projekt Ecochamps, in dem fünf Hybridfahrzeuge aufgebaut werden, um Standardisierungen bei Antrieb und Energiemanagement über verschiedene Fahrzeugsegmente hinweg zu demonstrieren: vom Pkw über den Lieferwagen bis hin zur Sattelzugmaschine. Die MTZ 10 behandelt übrigens das Ecochamps-Projekt aus der Perspektive der Antriebskomponenten. Nicht weniger interessant sind der Beitrag über Schwungmassenspeicher für Omnibusse sowie das Interview mit Dr. Carsten Intra, MAN, in dem auch die Ergebnisse des Gigaliner-Tests und die Zukunft von Car-to-X bei Nutzfahrzeugen thematisiert werden.

Die neuen, auf hoch- und vollautomatisiertes Fahren abzielenden Assistenzsysteme bringen die derzeit vorherrschenden E/E-Architekturen an ihre Grenzen. Funktionen für mittelschwere und schwere Nutzfahrzeuge wie Stau- und Autobahnpilot oder Pairing/Platooning setzen redundante Kommunikationskonzepte und Systemfunktionen voraus, stellen gleichzeitig aber auch erhöhte Anforderungen an die Stromversorgung im Fahrzeug. Eine zukunftsfähige E/E-Architektur muss zwingend innovative Funktionen wie etwa automatisiertes und elektrifiziertes Fahren, Cyber Security oder ein prädiktives Energiemanagement unterstützen.

Es deutet vieles darauf hin, dass der Nutzfahrzeugsektor der Enabler beim automatisierten Fahren sein könnte, da hier neben der Verkehrssicherheit die Total Cost of Ownership ein wesentlicher Treiber für eine zügige Entwicklung und
Einführung dieser Technik ist. Zudem erleichtert das im Vergleich zu Pkw eher berechenbare Fahrverhalten von Langstrecken-Nfz die Connectivity unter den Fahrzeugen. Der Gesetzgeber hat bereits erste Schritte unternommen: Der Ersatz konventioneller Rückspiegel durch Kamerasysteme wird in Kürze erlaubt sein. Dies beinhaltet bei Nutzfahrzeugen ein enormes Potenzial, insbesondere in Bezug auf die Sicherheit, da die menschliche Wahrnehmung durch diese Kamerasysteme unzweifelhaft optimiert werden kann. Das Display eines Kamera-MonitorSystems schafft weitgehende Möglichkeiten, dem Fahrer die notwendige Information über das Straßengeschehen dann zu liefern, wenn er sie benötigt.

Vor diesem Hintergrund wird es spannend sein zu sehen, welche Impulse die IAA Nutzfahrzeuge in diesem Jahr setzen kann.

Herzliche Grüße,

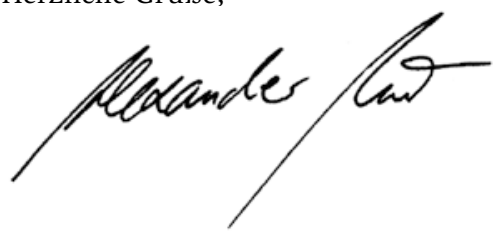

Dr. Alexander Heintzel Chefredakteur

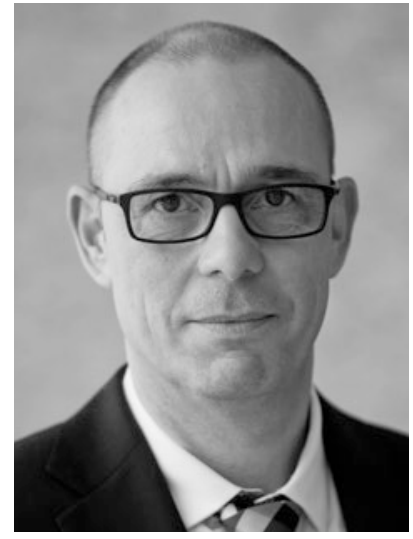

Wir

entwickeln, was bewegt.

IAV - Ihr Partner

für Automotive Engineering 